\title{
Perfil epidemiológico de adolescentes mujeres con antecedentes de agresión sexual consultantes en el área de salud mental de un centro de salud sexual y reproductiva
}

\author{
Carolina Leyton M., MSc ${ }^{1 a}$, Daniela Quezada $R^{2}$, Temístocles Molina G., MSc ${ }^{1 b}$ \\ 1 Centro de Medicina Reproductiva y Desarrollo Integral de la Adolescencia (CEMERA), Facultad de Medicina, \\ Universidad de Chile. ${ }^{2}$ Servicio Médico Legal. Santiago, Chile.
}

a Psicóloga. ${ }^{b}$ Estadístico.

\section{RESUMEN}

Objetivo: Conocer la frecuencia y describir las características de las agresiones sexuales infantiles en adolescentes mujeres ingresadas a la unidad de salud mental de un Centro de Salud Sexual y Reproductiva Adolescente. Método: Estudio de diseño transversal y analítico, se revisaron las fichas clínicas entre enero de 2006 y diciembre de 2009. Las variables estudiadas fueron: edad de la agresión, tipo y frecuencia de ésta; sexo, edad y tipo de relación con el agresor; revelación y persona a la que se le informa de la agresión sexual y denuncia de la agresión. Resultados: La mayor frecuencia de agresión sexual se presenta en la edad pre-escolar $(28,1 \%$ ) y la más común es el abuso sexual $(73,8 \%)$, tipo de agresión que es la más común en todas las edades. La mayoría de las agresiones fue un episodio único $(59,3 \%)$. La totalidad de los agresores eran conocidos o familiares de la víctima, todos varones, el 29,1\% eran menores de 18 años. El 36\% nunca reveló la agresión sufrida. El 31,8\% de las agresiones sin revelar ocurrieron en la edad preescolar. Sólo un $14,1 \%$ realizó denuncia. Conclusión: La mayoría de las agresiones sexuales ocurridas durante la infancia y adolescencia no son reveladas, ni denunciadas legalmente, manteniendo un alto nivel de silenciamiento y dificultades por parte de los equipos de salud para apoyar y tratar a las víctimas.

\section{PALABRAS CLAVE: Abuso sexual infantil, agresión sexual, infancia, adolescencia}

\section{SUMMARY}

Objective: To describe the frequency and characteristic of child sexual aggression in adolescent women admitted to the Mental Health Unit of an Adolescent Sexual and Reproductive Health Centre. Method: This is a cross-sectional and analytical study, clinical records were reviewed the between January 2006 and December 2009. The variables studied were: age of aggression, type and frequency of this aggression, sex, age and type of relationship with the perpetrator; disclosure and person who is informed and judicial complaint of sexual aggression. Results: The highest frequency of sexual aggression occurs in the preschool age $(28.1 \%)$ and the most common is sexual abuse $(73.8 \%)$, type of aggression is the most common in all ages. Most of the attacks were a single episode (59.3\%). All the attackers were males and acquaintances or relatives of victims, $29.1 \%$ were under 18 years old. $36 \%$ never disclosure the assault. The $31.8 \%$ of unveiled attacks occurred in preschool. Only $14.1 \%$ made a judicial complaint. Conclusion: The majority of sexual assaults that occurred during childhood and adolescence are unveiled, nor legally reported maintaining a high level of silencing and difficulties by health equipment to support and treat victims.

\section{KEY WORDS: Child sexual abuse, sexual aggression, childhood, adolescence}




\section{INTRODUCCIÓN}

El abuso sexual infantil corresponde al involucramiento de menores de edad en actividades sexuales no acordes a su nivel cognitivo, emocional o social (1). Legalmente, se define como actos de significación sexual distintos a la penetración (2).

La prevalencia descrita de agresiones sexuales es variable, dado que se utilizan definiciones y metodologías distintas en cada estudio, además entre 75 y $80 \%$ de ellas no son denunciadas, dificultando determinar su prevalencia real (3-10).

Antes de los 18 años, entre 12 y $25 \%$ de las mujeres ha sido víctima de agresiones sexuales $(11,12)$. De las que asisten a un servicio de urgencia, 43\% son adolescentes (13). En escolares chilenos, 8,25\% había sufrido alguna agresión sexual (14). En población consultante en salud mental la prevalencia aumenta y un $42 \%$ presenta antecedentes de contacto sexual forzado infantil (15-17).

Las agresiones sexuales infantiles repercuten psicológicamente sobre el desarrollo de las víctimas, además de sufrir consecuencias físicas, emocionales, sociales y familiares $(3-5,12,16,18-20)$. Existe asociación entre las agresiones sexuales infantiles y el aumento del riesgo de trastornos psiquiátricos $(3,4,16,19,21-24)$, y consecuencias en la salud mental en la vida adulta. Además, muchas agresiones no son reveladas hasta años después de ocurrido el episodio $(10,16,17)$, lo que nos deja la inquietud de atenciones en salud mental que pudieran tener oculta una vivencia de agresión sexual en la infancia.

Considerando que parte importante de la población consultante a salud mental puede presentar el antecedente de agresión sexual infantil y las repercusiones que éste provoca en la salud de las víctimas, este trabajo tiene como propósito conocer la frecuencia y describir las características de las distintas agresiones sexuales sufridas por adolescentes consultantes en el área de salud mental de un centro de Salud Sexual y Reproductiva.

Se pretende contribuir al conocimiento de las diferentes configuraciones de las agresiones sexuales, apoyando la visualización y comprensión del fenómeno, ayudando a su pesquisa y diagnóstico por parte de los equipos de salud, aportando al apoyo del tratamiento de las víctimas, la prevención en el ambiente familiar y la implementación de programas de prevención.

\section{PACIENTES Y MÉTODO}

Estudio de diseño transversal y analítico. Universo de estudio constituido por adolescentes de sexo femenino, menores de 18 años, que consultaron en el Centro de Medicina Reproductiva y Desarrollo Integral de la Adolescencia (CEMERA) de la Facultad de Medicina de la Universidad de Chile, que fueron referidas a la Unidad de Salud Mental y que reportaron el antecedente de haber sufrido una agresión sexual. Se excluyeron del estudio a los adolescentes masculinos. Se realizó una revisión de las fichas clínicas de salud mental, entre enero de 2006 y diciembre de 2009. Las adolescentes al momento del ingreso a CEMERA firmaron un consentimiento informado donde se les explicó que los datos podrían ser utilizados en investigación protegiendo sus identidades.

Las variables estudiadas fueron: edad $y$ etapa del desarrollo de la agresión sexual (preescolar: cinco a seis años; escolar: siete a nueve años; adolescencia inicial: diez a doce años, y adolescencia media: 13 a 16 años). Los tipos de agresión sexual fueron definidas según las leyes nacionales de delitos sexuales № 19.617 (26) y № 19.927 (2): Violación: acceso carnal por vía vaginal, anal o bucal a un menor de 14 años o cuando se usa fuerza o intimidación, víctima privada de sentido o incapaz de oponer resistencia o cuando se abusa de un trastorno mental. Abuso sexual: corresponde a todo acto de significación sexual relevante diferente al acceso carnal, que haya afectado los genitales, ano o boca, existiendo o no contacto directo con la víctima y concurriendo las mismas circunstancias que la violación. En cuanto al intento de violación en esta investigación fue definido por las adolescentes como una intimidación física a tener actividad sexual. Frecuencia de la agresión sexual, descrita como un evento único o reiterado; sexo, edad y relación de la víctima con el agresor; contacto actual con el agresor sexual, describiendo si la ausencia de contacto es por una medida de protección tomada hacia la víctima; revelación de la agresión sexual; momento en que ésta ocurre, inmediata o tardía (más de un año) y a quién; existencia de una denuncia judicial. Se realizaron análisis uni y bivariado, aplicándose las pruebas estadísticas de Chi2 para una muestra y test Kruskal-Wallis, se utilizaron software Excel y STATA 10.0 .

\section{RESULTADOS}

La Unidad de Salud Mental de CEMERA realizó un total de 293 ingresos entre 2006 y 2009 . Los resultados corresponden al $21,8 \%(n=64)$ de pacientes de sexo femenino que presentaban el antecedente de haber sufrido una agresión sexual. Estas adolescentes provienen de las diferentes comunas de la Región Metropolitana. El nivel socioeconómico fue medio-bajo, la mediana de edad al ingreso fue de 16 años de edad (rango: 6-18 años).

El $15,6 \%(n=10)$ presentó en otro momento de su vida una segunda agresión sexual perpetrada por otro individuo distinto al de la primera agresión. La mediana de la edad de la primera agresión sexual fue de 9 años (rango: 5-16 años). La eta- 
pa del desarrollo de mayor frecuencia de agresión sexual corresponde a pre-escolar $(28,1 \%)$, seguida de escolar $(25 \%)$ y adolescencia media $(25 \%)$, cifras que no muestran diferencias significativas.

En cuanto al tipo de agresión sexual, mostró diferencias significativas $(p=0,001)$, la más frecuente fue el abuso sexual $(73,8 \%)$, seguido de violación vía vaginal $(13,1 \%)$, intentos de violación $(8,2 \%)$ y violación vía oral $(4,9 \%)$.

Al relacionar la etapa del desarrollo en que ocurrió la agresión sexual y el tipo de agresión sufrida, se encontró en todos los rangos de edad el abuso sexual como agresión sexual más común $(69,2 \%$ a $87,6 \%$ ) (Tabla I). Por otro lado, en cuanto a las violaciones por vía vaginal $(n=8)$ el mayor porcentaje ocurrió en la adolescencia media $(62,5 \%)$ y en la adolescencia inicial (25\%).

En cuanto a la duración de la agresión sexual, del total de adolescentes que refirió el tiempo de la agresión sexual y el tipo de agresión, el 59,3\% presentó un episodio único de agresión sexual y $40,7 \%$ refirió agresión reiterada. Se destaca el caso de violaciones vaginales $(n=8)$ en que el $87,5 \%$ fueron eventos únicos.

La totalidad de los agresores sexuales eran conocidos o familiares de la víctima. Un $26,6 \%$ conocidos como vecinos, amigos de su hermano o de la familia. Le siguen los primos $(20,3 \%)$, padre o padrastro $(17,2 \%)$ y tíos $(14,1 \%)$. Además, existen otros tipos de agresores sexuales relacionados directamente con la menor, como el abuelo, cuñado o un amigo, que corresponden a un porcentaje no menor de los agresores $(21,9 \%)$.

En relación al agresor sexual en este estudio, todos fueron hombres, destacándose que el $29,1 \%$ de éstos fueron menores de 18 años. La mediana de edad del agresor fue 30 años (rango: 9-70 años).

De las agresiones únicas el 30,6\% fue cometida por un conocido, y en el caso de las reiteradas el $26,9 \%$ fue perpetrada por un primo de la menor. Un $36 \%$ de las niñas nunca reveló la agresión sexual sufrida. Del resto, $41 \%$ lo hizo antes de un año y $23 \%$ reveló después de un año de ocurrida la agresión, el único tipo de agresión sexual que siempre fue revelado fue la violación vaginal. Al asociar las variables revelación de la agresión sexual y el tipo de agresión, del total de niñas sin revelar el $85 \%$ había presentado una agresión sexual del tipo abuso sexual (Tabla II).

Las adolescentes que revelaron de manera inmediata, tienen mayor edad que las que lo realizan de manera tardía $(p=0,001)$ o no revelan $(p=0,01)$. No hubo diferencias significativas en la edad de la agresión entre las que no revelaron la agresión y las que lo hacen tardíamente (Tabla III).

El $50 \%$ de las agresiones únicas fue revelado inmediatamente. Un $31,8 \%$ de las agresiones sexuales sin revelación ocurrieron en la etapa pre-escolar. Entre los abusos reiterados, un porcentaje importante $(38,5 \%)$ quedó sin revelación. Se encontraron diferencias significativas en relación a quien se revelaba la agresión sexual sufrida $(p=0,01)$; más de la mitad $(55,3 \%)$ refirió el episodio de agresión a su madre, seguido por algún familiar $(26,3 \%)$ y sólo la minoría a un miembro fuera del

Tabla I

TIPO DE AGRESIÓN SEXUAL Y ETAPA DEL DESARROLLO

\begin{tabular}{lccccc}
\hline $\begin{array}{l}\text { Etapa del desarrollo } \\
\text { 1a agresión sexual }\end{array}$ & $\begin{array}{c}\text { Abuso sexual } \\
\mathrm{n}(\%)\end{array}$ & $\begin{array}{c}\text { Vía oral } \\
\mathrm{n}(\%)\end{array}$ & $\begin{array}{c}\text { Vía vaginal } \\
\mathrm{n}(\%)\end{array}$ & $\begin{array}{c}\text { Intento de violación } \\
\mathrm{n}(\%)\end{array}$ & $\begin{array}{c}\text { Total } \\
\mathrm{n}(\%)\end{array}$ \\
\hline Preescolar & $12(70,6)$ & $1(5,9)$ & $1(5,9)$ & $3(17,6)$ & $17(100)$ \\
Escolar & $14(87,6)$ & $1(6,2)$ & $0(0,0)$ & $1(6,2)$ & $16(100)$ \\
Adolescencia inicial & $9(69,2)$ & $1(7,7)$ & $2(15,4)$ & $1(7,7)$ & $13(100)$ \\
Adolescencia media & $10(66,7)$ & $0(0,0)$ & $5(33,3)$ & $0(0,0)$ & $15(100)$ \\
\hline
\end{tabular}

Tabla II

TIPO DE AGRESIÓN Y MOMENTO DE REVELACIÓN

\begin{tabular}{lccccc}
\hline Momento de revelación & $\begin{array}{c}\text { Abuso sexual } \\
\mathrm{n}(\%)\end{array}$ & $\begin{array}{c}\text { Vía oral } \\
\mathrm{n}(\%)\end{array}$ & $\begin{array}{c}\text { Vía vaginal } \\
\mathrm{n}(\%)\end{array}$ & $\begin{array}{c}\text { Intento de violación } \\
\mathrm{n}(\%)\end{array}$ & $\begin{array}{c}\text { Total } \\
\mathrm{n}(\%)\end{array}$ \\
\hline Inmediata & $18(75,0)$ & $0(0,0)$ & $5(20,8)$ & $1(4,2)$ & $24(100)$ \\
Tardía & $7(50,0)$ & $2(14,3)$ & $3(21,4)$ & $2(14,3)$ & $14(100)$ \\
Sin revelación & $17(85,0)$ & $1(5,0)$ & $0(0,0)$ & $2(10,0)$ & $20(100)$ \\
\hline
\end{tabular}


Tabla III

\section{EDAD DE LA AGRESIÓN Y MOMENTO DE LA REVELACIÓN}

\begin{tabular}{lccc}
\hline & \multicolumn{3}{c}{ Momento de la revelación } \\
$\begin{array}{l}\text { Edad de la } \\
\text { agresión }\end{array}$ & Inmediata & Tardía & Sin revelación \\
\hline Años \pm DE & $11,1 \pm 3,1$ & $7,8 \pm 2,2$ & $8,9 \pm 3,3$ \\
\hline
\end{tabular}

núcleo familiar (18,4\%).

De todas las agresiones sexuales, sólo $14,1 \%$ $(n=9)$ fueron denunciadas judicialmente, lo que muestra diferencias significativas frente al resto $(p=0,001)$. De las denuncias realizadas, $44,4 \%$ correspondían a violación vaginal. Del total de menores que no denunciaron $(n=52) 80,8 \%$ había sufrido abusos sexuales (Tabla IV).

Presenta en la actualidad algún contacto con su agresor sexual el $41,3 \%$ de las víctimas $(n=19)$, de ellas, el agresor más frecuente fue el primo $(31,6 \%)$. No tienen contacto con el agresor $58,7 \%$ de las víctimas, de ellas, $14,8 \%$ debido a razones independientes a la agresión sexual, como cambio de domicilio de la víctima y/o su familia.

\section{DISCUSIÓN}

La frecuencia de mujeres adolescentes que consultan a salud mental y que presentan el antecedente de una agresión sexual $(21,8 \%)$ es inferior a lo reportado por Perales y cols $(28,9 \%)(15)$. Asimismo, coincide con lo establecido en la literatura, como prevalencia de sufrir una agresión sexual durante la vida de la población general femenina, y que oscila entre 15 y $25 \%$ (5), también con lo descrito por la OMS (27) en relación a que la iniciación sexual forzada de las adolescentes en algunas localidades del mundo supera el 30\% (19).

Cabe recalcar la importancia de la sub-notificación de las denuncias debido al silenciamiento de las víctimas y sus familias, lo que dificulta que las mujeres puedan ser apoyadas y atendidas en las problemáticas sociales, psicológicas, emocionales y familiares que se presentan como consecuencia de la agresión $(9,28)$, lo que dificulta la comprensión y real dimensión de las consecuencias de las agresiones sexuales.

La edad más frecuente de las agresiones sexuales que se encontró en este estudio coinciden con lo descrito en la literatura nacional e internacional, evidenciando que la edad pre-escolar y escolar son claves para desarrollar programas de prevención y apoyo a las víctimas de agresiones sexuales, además de otorgar la oportunidad de revelar las situaciones abusivas que se puedan estar viviendo $(3,4,6,11,29)$.

El Servicio Médico Legal en sus estadísticas del año 2010 (29), describe que la agresión sexual más común fue el abuso sexual, lo que coincide con esta investigación. Por otro lado, en la literatura internacional se señala que de los diferentes tipos de violación que puede sufrir una adolescente, predomina la violación vía vaginal, lo que también concuerda con los resultados presentados (13).

Este estudio muestra que la mayoría de las violaciones vaginales fueron eventos únicos, lo que se explica porque este tipo de agresión se cometió en niñas de mayor edad y corresponden a un evento traumático de mayor intensidad que puede ser verbalizado y revelado más que en las niñas menores (3-6-28).

La totalidad de los agresores sexuales en este estudio fueron hombres, familiares o conocidos de las víctimas, ambos aspectos coinciden con los resultados de la literatura nacional e internacional $(3,6,8,10,11)$.

Más de un tercio de las mujeres de este estudio no le cuenta a nadie la agresión sexual sufrida. Es importante destacar que a mayor edad, las adolescentes revelan más prontamente la agresión sexual sufrida, lo que también es concordante con lo descrito en relación a que a menor edad, existe más confusión respecto a la interacción abusiva con el agresor cercano, el temor a que no se crea en su relato y al proceso de secreto que implica la situa-

Tabla IV

TIPO DE AGRESIÓN SEXUAL Y DENUNCIA JUDICIAL

\begin{tabular}{cccccc}
\hline Denuncia & $\begin{array}{c}\text { Abuso sexual } \\
\mathrm{n}(\%)\end{array}$ & $\begin{array}{c}\text { Vía oral } \\
\mathrm{n}(\%)\end{array}$ & $\begin{array}{c}\text { Vía vaginal } \\
\mathrm{n}(\%)\end{array}$ & $\begin{array}{c}\text { Intento de violación } \\
\mathrm{n}(\%)\end{array}$ & $\begin{array}{r}\text { Total } \\
\mathrm{n}(\%)\end{array}$ \\
\hline $\mathrm{Si}$ & $3(33,3)$ & $0(0,0)$ & $4(44,4)$ & $2(22,2)$ & $9(100)$ \\
$\mathrm{No}$ & $42(80,8)$ & $3(5,8)$ & $4(7,7)$ & $3(5,8)$ & $52(100)$ \\
\hline
\end{tabular}


ción de abuso sexual (28).

El estudio muestra que un porcentaje bajo de las agresiones sexuales hacia las adolescentes fueron denunciadas a la justicia, lo que concuerda con lo encontrado en otros estudios nacionales, que describen diversas razones para que la víctima o su familia no realicen la denuncia, como la dependencia de la víctima con el agresor, amenaza del agresor, temor a la reacción de los padres, bajo índice de esclarecimiento del delito, entre otras $(4,9,29)$.

Esta investigación presenta limitaciones, dado que es un estudio con una muestra sesgada y pequeña, sin embargo, podemos concluir que los resultados se corresponde con lo descrito en la literatura, y puede colaborar al tratamiento de pacientes que consultan a salud mental de manera repetida y constante. Por otro lado, los antecedentes son de carácter retrospectivo, por lo que el recuerdo puede influir en la información entregada por las pacientes.

\section{CONCLUSIÓN}

Este estudio muestra el perfil epidemiológico de las víctimas de agresiones sexuales en pacientes que consultan a una unidad de salud mental de un centro de Salud Sexual y Reproductiva especialista en adolescencia. Podemos describir que las agresiones sexuales ocurrieron con mayor frecuencia en la etapa pre-escolar, el principal tipo de agresión sufrida es el abuso sexual y el agresor es un familiar o un conocido de su familia. Las agresiones sexuales habitualmente no se revelan o se hace muy tardíamente, y con menor frecuencia se realiza una denuncia formal a la justicia. Todos estos factores contribuyen a que las agresiones sexuales continúen siendo un tema sub-notificado, de difícil evaluación y que muchos de estos casos queden impunes. Dadas las limitaciones del estudio, las conclusiones deben ser consideradas como una aproximación descriptiva y analítica al estudio de la frecuencia y diferentes configuraciones de las agresiones sexuales, en adolescentes mujeres consultantes a salud mental.

\section{REFERENCIAS}

1. MINSAL-UNICEF. Guía Clínica. Atención niños niñas y adolescentes, menores de 15 años víctimas de abuso sexual. Santiago, Mayo 2011. Hallado en: http:// www.minsal.gob.cl/portal/url/item/aaa27720f363a745 e04001011e011120.pdf

2. Ley $N^{\circ}$ 19.927: Modifica el código penal, el código de procedimiento penal y el código procesal penal en materia de delitos de pornografía infantil. Diario Oficial de la República de Chile. Miércoles 14 de enero 2004.

3. Espinoza M. Consecuencias en el desarrollo del abuso sexual en la adolescencia. En: Valdivia M, Condeza M, Editores. Psiquiatría del adolescente. Santiago, Chile: Editorial Mediterráneo Ltda.; 2006. pp 383-95.

4. González E. Abusos sexuales en adolescentes: factores predictivos de riesgo. En: Molina R, Sandoval J, González E, Editores. Salud Sexual y Reproductiva de la Adolescencia. Santiago, Chile: Editorial Mediterráneo Ltda.; 2003. pp 610-20.

5. MINSAL. Normas y guía clínica para la atención en servicios de urgencia de personas víctimas de violencia sexual. Santiago, Chile. 2004. Hallado en: http:// www.infojoven.cl/documentos/normaViolencia.pdf

6. Álvarez K. Abuso sexual en la niñez y adolescencia En: Almonte C., Montt M., Correa A. Editores. Psicopatología infantil y de la adolescencia. Santiago, Chile: Editorial Mediterráneo; 2003 pp 486-506.

7. Molina R. Abuso sexual en adolescentes: aspectos epidemiológicos En: Molina R., Sandoval J., González E. Editores, Salud Sexual y Reproductiva de la Adolescencia. Santiago, Chile: Editorial Mediterraneo; 2003. pp 596-607.

8. Schönbucher V, Maier T, Held L, Mohler-Kuo M, Schnyder U, Landolt MA. Prevalence of child sexual abuse in Switzerland: a systematic review. Swiss Med Wkly 2011;140:13123.

9. Montero A, Cerda C. Principales necesidades de atención de las víctimas de delitos sexuales. Rev Sogia 2002;9:31-6.

10. Leserman J. Sexual abuse history: prevalence, health effects, mediators, and psychological treatment. Psychosomatic Medicine 2005;67:906-15.

11. Pereda N, Guilera G, Forns M, Gómez-Benito J. The International epidemiology of child sexual abuse: a continuation of Finkelhor (1994). Child Abuse Negl 2009;33:331-42.

12. Lahoti S, McClain N, Girardet, McNeese M, Kim C. Evaluating the child for sex abuse. Am Fam Physician 2001;63:883-92.

13. Jones J, Rossman L, Wynn B, Dunnuck C, Schwartz N. Comparative analysis of adult versus adolescent sexual assault: epidemiology and patterns oh anogenital injuries. Acad Emerg Med 2003;10:872-7.

14. Florenzano R, Pino P, Kaplan M, Burrows J. Frecuencia de maltrato infantil y sexual en escolares de Santiago de Chile: antecedentes familiares y consecuencias. Rev Psiquiatr 1992;12:60-6.

15. Perales P, Florenzano R, Rojas C, Feurhake H. Maltrato físico y abuso sexual: sus consecuencias psicopatológicas. Rev Psiquiat Clínica 1998;31:45-56.

16. Vitriol V, Gomberoff J, Basualto M, Ballesteros S. Relación entre estrés post-traumático de inicio tardío, abuso sexual infantil y revictimización sexual. Caso clínico. Rev Méd Chile 2006;134:1302-5.

17. Vitrol V. Relación entre psicopatología adulta y antecedentes de trauma infantil. Rev Chil Neuro-Psiquiat 2005;43:88-96.

18. Policía de Investigaciones de Chile. Centro de Asistencia a Víctimas de Atentados Sexuales CAVAS Metropolitano: 16 años de experiencia. Santiago, Chile. (2004).

19. Jhonson Felze C. Child sexual abuse. Lancet 
2004;364:462-70.

20. American Academy of Pediatrics, Comittee on Child Abuse and Neglect; Guidelines for the Evaluation of Sexual Abuse of Children: Subject Review. Pediatrics 1999;103:186-91.

21. Weil K, Florenzano R, Vitrol V, Cruz C, Carvajal C, Fullerton C, Muñiz C. Trauma infanto juvenil y psicopatología adulta: un estudio empírico. Rev Méd Chile 2004;132:1499-504.

22. Senn T, Carey M, Vanable P, Coury-Doniger P, Urban $M$. Characteristics of sexual abuse in childhood and adolescence influence sexual risk behavior in adulthood. Arch Sex Behav 2007;36:637-45.

23. Edwards VJ, Hokden GW, Felliti VJ, Anda RF. Relationship between multiple forms of childhood maltreatment and adult mental health in community respondents: Results from the adverse childhood experiences study. Am J Psychiatr 2003;160:1453-50.

24. Chen L, Murad H, Paras M, Colbenson K, Sattler A, Goranson E, et al. Sexual abuse and lifetime diag- nosis of psychiatric disorders: systematic review and meta-analysis. Mayo Clin Proc 2010;85:618-29.

25. Spataro J, Mullen P, Burguess P, Wells D. Moss S. Impact of child sexual abuse on mental health: prospective study in males and females. Br J Psychiatry 2004; 186:416-42.

26. Ley $\mathrm{N}^{\circ}$ 19.617: Modifica el código penal, el código de procedimiento penal y otros cuerpos legales en materias relativas al delito de violación. Diario Oficial de la República de Chile. Lunes 12 de julio de 1999.

27. WHO. Sexual Violence. En: Etienne G., Linda L., James A. Anthony B. and Rafael Lozano. Editores, World Report on Violence and Health. Geneva: Editorial WHO; 2002. pp 148-81.

28. Roma E, Moya G. Aspectos generales y psicopatológicos del abuso sexual en niños. Rev Chil Pediatr 1989;80:71-5.

29. Nahuelpán E, Insunza J. Unidad de Estadísticas y Archivo Médico Legal. La violencia de género en Chile período 2000 - 2010. Santiago, Abril 2011. 